\title{
On the recoil and Doppler shifts
}

\author{
Stephen M. Barnett * \\ Department of Physics, SUPA, University of Strathclyde, Glasgow G4 0NG, UK \\ and Department of Physics, FNSPE, Czech Technical University in Prague, Břehová 7, 11519 Praha 1 , \\ Czech Republic
}

(October 2009)

\begin{abstract}
The recoil shift due to the finite mass of an absorbing atom is, when viewed in the right way, simply part of the total Doppler shift. This
\end{abstract} is true both in the low speed and relativistic limits and also for the reflection of light from a low-mass mirror.

\section{Introduction}

The absorption of a photon by an atom changes its internal state, but also modifies its kinetic energy and momentum. This phenomenon, of course, underlies the field of laser cooling. The recoil shift is an addition to the familiar Doppler shift, which accounts for this effect [1]. This shift is typically in the kHz region and, although small, has been measured [2].

Our purpose, in this paper, is to suggest that the recoil shift is, in fact, part of the Doppler shift, but that the correct velocity is not that of the atom before the absorption, but rather the arithmetical average of this initial velocity and its value after the absorption.

Our analysis relies only on the conservation of energy and momentum, and is readily extended to the relativistic domain. We show that the required photon frequency for resonant absorption is then arithmetical average of the Doppler shifts associated with the velocity of the atom before and after the absorption.

We conclude with a derivation of the Doppler shift relevant to the reflection of a photon from a low-mass moving mirror. I this case we find, surprisingly, that it is the geometric average of the Doppler shifts for the initial and final mirror velocities that matters.

\section{Absorption of a photon by a moving atom}

We consider the absorption of a photon by an atom moving towards the light source. As is well known, the frequency of the light, as it appears to the atom, is increased due to the Doppler shift. If the atom is moving with velocity $v$ then the frequency in the atom's frame $\omega_{a}$ is related to that in the laboratory frame, $\omega$ by

$$
\omega_{a}=\omega\left(1+\frac{v}{c}\right)
$$

If the atom is moving very rapidly, then we need the to use the formula

$$
\omega_{a}=\omega \mathcal{D}(v)
$$

\footnotetext{
${ }^{*}$ Corresponding author. Email: steve@phys.strath.ac.uk

Journal of Modern Optics

ISSN 0950-0340 print/ISSN 1362-3044 online (c) 200x Taylor \& Francis

http://www.tandf.co.uk/journals

DOI: $10.1080 / 0950034 Y Y x x x x x x x x$
} 
where $\mathcal{D}(v)$ is the relativistic Doppler factor

$$
\mathcal{D}(v)=\sqrt{\frac{1+\frac{v}{c}}{1-\frac{v}{c}}}
$$

Clearly, for small velocities, this reduces to the non-relativistic expression (2).

Fermi showed that we can derive the non-relativistic Doppler formula simply by imposing the conservation of energy and momentum on the emission of a photon [3]. It is instructive to repeat Fermi's calculation and we do so, but consider absorption rather than emission and specialize to a single spatial dimension. Let an atom of mass $m$ be moving with velocity $v$ towards a light source. Consider an optical transition at angular frequency $\omega_{0}$, and let the light have angular frequency $\omega$ in the laboratory frame. The abosorption event will reduce the velocity of the atom and we let the velocity after absorption be $v^{\prime}$. Let us impose the conservation of energy and momentum:

$$
\begin{aligned}
\hbar \omega_{0}+\frac{1}{2} m v^{\prime 2} & =\hbar \omega+\frac{1}{2} m v^{2} \\
m v^{\prime} & =m v-\frac{\hbar \omega}{c} .
\end{aligned}
$$

Fermi's approach to these equations was to square the second one and neglect terms of order $c^{-2}$ to give

$$
m v^{\prime 2}=m v^{2}-2 v \frac{\hbar \omega}{c} .
$$

Substituting this into (4) then gives the Doppler formula for resonance

$$
\omega_{0}=\omega\left(1+\frac{v}{c}\right) .
$$

It is not necessary, of course, to neglect terms of order $c^{-2}$ and we can restore the term omitted in Fermi's approximation by replacing (6) with

$$
m v^{2}=m v^{2}-2 v \frac{\hbar \omega}{c}+\frac{1}{m}\left(\frac{\hbar \omega}{c}\right)^{2} .
$$

If we insert this into our energy conservation equation (4) then we obtain a resonant condition in which the Doppler shift is supplemented by an additional small shift, which is the recoil shift [1]

$$
\omega_{0}=\omega\left(1+\frac{v}{c}-\frac{\hbar \omega}{2 m c^{2}}\right) .
$$

We can see that the recoil shift is small, being the frequency multiplied by the ratio of the energy of the photon to twice the rest-mass energy of the atom.

Let us consider the effect of eliminating the mass rather than the final velocity from our energy and momentum equations (4) and (5). To achieve this, we first rewrite the equations in the form

$$
\begin{aligned}
\frac{1}{2} m v^{\prime 2}-\frac{1}{2} m v^{2} & =\hbar \omega-\hbar \omega_{0} \\
m v^{\prime}-m v & =-\frac{\hbar \omega}{c},
\end{aligned}
$$


and then divide the first equation by the second to obtain

$$
\begin{aligned}
\frac{v^{\prime}+v}{2 c} & =\frac{\omega-\omega_{0}}{-\omega} \\
\Rightarrow \quad \omega_{0} & =\omega\left(1+\frac{v^{\prime}+v}{2 c}\right) .
\end{aligned}
$$

The recoil shift has been absorbed into the Doppler shift, but the relevant velocity is $\left(v^{\prime}+v\right) / 2$, that is the mean, or arithmetical average of the velocities before and after the absorption.

\section{Relativistic treatment for a rapidly moving atom}

We might ask whether the (total) Doppler shift in (12) is the average of the Doppler shifts at velocities $v$ and $v^{\prime}$ or, perhaps, the Doppler shift at the average velocity $\left(v^{\prime}+v\right) / 2$. At low velocities, in the nonrelativistic limit, there is no difference between these. To address the question, therefore, we need to perform a relativistic analysis. We let $m$ be the rest mass and introduce the familiar Lorentz factor

$$
\gamma=\frac{1}{\sqrt{1-\frac{v^{2}}{c^{2}}}},
$$

with a similar expression for the factor, $\gamma^{\prime}$, after the absorption. Our expressions for the conservation of energy and momentum (4) and (5) become

$$
\begin{aligned}
\gamma^{\prime}\left(m c^{2}+\hbar \omega_{0}\right) & =\gamma m c^{2}+\hbar \omega \\
\gamma^{\prime}\left(m+\frac{\hbar \omega_{0}}{c}\right) v^{\prime} & =\gamma m v-\frac{\hbar \omega}{c} .
\end{aligned}
$$

Proceeding as before, we can eliminate the mass to obtain the effective form of the Doppler shift:

$$
\begin{aligned}
\frac{\omega_{0}}{\omega} & =\frac{\gamma(c+v)-\gamma^{\prime}\left(c+v^{\prime}\right)}{\gamma \gamma^{\prime}\left(v-v^{\prime}\right)} \\
\Rightarrow \omega_{0} & =\omega \frac{2}{D(-v)+D\left(-v^{\prime}\right)} .
\end{aligned}
$$

In the relativistic regime, the required frequency of the field for resonant excitation of the atom is the arithmetic mean of the Doppler-shifted transition frequencies before and after the absorption:

$$
\omega=\omega_{0} \frac{D(-v)+D\left(-v^{\prime}\right)}{2} .
$$

For velocities in the non-relativistic regime this reduces to the simple form

$$
\omega=\omega_{0}\left(1-\frac{v^{\prime}+v}{2 c}\right)
$$

as it should. 


\section{Reflection from a low-mass mirror}

It is a well-known consequence of the Doppler effect that the frequency of light is changed if it is reflected from a moving mirror. Let us consider a single photon, with angular frequency $\omega$, and a mirror travelling towards the source of the photon will velocity $v$. The frequency of the reflected photon, $\omega^{\prime}$, will then be greater than that of the incident photon, with the frequency up-shifted by the factor [4]

$$
\frac{\omega^{\prime}}{\omega}=\frac{1+\frac{v}{c}}{1-\frac{v}{c}}=\mathcal{D}^{2}(v)
$$

Let us suppose that the mirror is of sufficiently low mass, $m$, that the reflection of the photon can change its velocity from $v$ to $v^{\prime}$ and impose on the mirror and the photon the conservation of energy and momentum. We find that

$$
\begin{aligned}
\gamma^{\prime} m c^{2}+\hbar \omega^{\prime} & =\gamma m c^{2}+\hbar \omega \\
\gamma^{\prime} m v^{\prime}+\frac{\hbar \omega^{\prime}}{c} & =\gamma m v-\frac{\hbar \omega}{c}
\end{aligned}
$$

Eliminating, once more, the mass we find that the ratio of the frequencies is

$$
\begin{aligned}
\frac{\omega^{\prime}}{\omega} & =\frac{\gamma(c+v)-\gamma^{\prime}\left(c+v^{\prime}\right)}{-\gamma(c-v)+\gamma^{\prime}\left(c-v^{\prime}\right)} \\
& =\mathcal{D}(v) \mathcal{D}\left(v^{\prime}\right)
\end{aligned}
$$

If we compare this with the high-mass mirror expression (19) then we see that the relevant Doppler factor here is the geometric mean of those for the initial and final velocities of the mirror.

We should investigate the limiting form of this Doppler shift in the non-relativistic regime. For small velocities, the ratio of frequencies (22) becomes

$$
\frac{\omega^{\prime}}{\omega} \approx\left(1+\frac{v}{c}\right)\left(1+\frac{v^{\prime}}{c}\right) \approx 1+\frac{v+v^{\prime}}{c}
$$

which is the Doppler shift for the sum of the mirror velocities before and after the reflection. Remarkably, we can interpret the relativistic expression in the same way if we use the relativistic velocity-addition formula so that the sum of the two velocities is

$$
V=\frac{v+v^{\prime}}{1+\frac{v v^{\prime}}{c^{2}}}
$$

The ratio of the frequencies of the reflected and incident photons is then

$$
\frac{\omega^{\prime}}{\omega}=\mathcal{D}(v) \mathcal{D}\left(v^{\prime}\right)=\mathcal{D}(V)
$$

which is the relativistic Doppler shift at the (relativistic) sum of the velocities.

\section{Conclusions}

Fermi showed that the Doppler shift can be determined simply by applying the conservation of energy and momentum to a radiative process [3]. His analysis provides, also, the recoil shift which supplements the Doppler shift. We have shown that the total shift is perhaps more correctly viewed as simply a Doppler 
shift, but that the relevant velocity is the average of the absorbing body before and after the absorption takes place.

We have shown that Fermi's analysis can easily be extended into the relativistic regime in which atom is moving at high velocity. In this case, the total frequency shift is again a Doppler shift: the required optical frequency for resonant absorption is given by the arithmetic mean of the Doppler shifts for the velocities before and after the absorption.

Finally, we have applied the same reasoning to the problem of reflection of a photon from a moving mirror. We have considered the mirror to be of sufficiently low mass that the reflection of a single photon makes a change to its velocity. In this case we found that the total frequency shift is again a Doppler shift, given by $\mathcal{D}^{2}$, with the relevant shift being the geometric mean of those for the initial and final velocities of the mirror:

$$
\mathcal{D}=\sqrt{\mathcal{D}(v) \mathcal{D}\left(v^{\prime}\right)} .
$$

In this case, moreover, the nett shift can be interpreted simply as the familiar Doppler shift, given by $\mathcal{D}$, with the relevant velocity being the relativistic sum of the initial and final velocities.

In each case the mass of the atom or mirror makes no appearance and it is clear that, by identifying the relevant velocity, the recoil shift should be thought of as nothing more than part of the Doppler shift.

\section{Acknowledgements}

I thank Paul Lett, Peter Milonni and Erling Riis for helpful comments and suggestions. I also thank Igor Jex for his hospitality during my visit to Prague during which I had the time to complete this project. This work was supported by the projects MSM 684077039 and LC06002 of the Czech Republic, by the Royal Society and by the Wolfson Foundation.

\section{References}

[1] S. Stenholm, Rev. Mod. Phys. 58, 699-739 (1986).

[2] J. L. Hall, C. J. Bordé and K. Uehara, Phys. Rev. Lett. 37, 1339-1342 (1976).

[3] E. Fermi, Rev. Mod. Phys. 4, 87-132. (1932).

[4] W. H. McCrea Relativity Physics Fouth Edition (Methuen and Co., London, 1954). 